\title{
Cross-modal transfer in rats following different early environments
}

\author{
EDWARD H. YETERIAN and WILLIAM A. WILSON, JR. \\ University of Connecticut, Storrs, Connecticut 06268
}

\begin{abstract}
The present study investigated the effects of different early environments on specific cross-modal transfer (CMT) in rats, using a direct vs. reversal transfer paradigm. At weaning, littermates were divided into two groups: EC, reared in an enriched environment, and IC, reared in an impoverished environment. All animals remained in their respective environments until 96 days of age, at which time they were trained on a visual intensity discrimination. Upon completion of visual training, they received an auditory intensity discrimination (transfer). Both the EC and IC groups showed specific CMT; however, there was no difference between the two groups in the magnitude of the transfer effect. Furthermore, learning ability in the visual modality was equivalent for both groups. The results suggest that specific CMT of intensity is not affected by differential early environmental experience. Some possible explanations are discussed.
\end{abstract}

Studies of specific cross-modal transfer (CMT) determine whether information gained in one modality is used in responding to stimuli presented in an alternative modality. Over and Mackintosh (1969) have demonstrated that rats trained to discriminate between high and low intensities of light, or of sound, have a significant tendency to respond in a similar manner (i.e., to high vs. low) when presented with stimuli differing along the intensity dimension in the alternative modality. In recent studies using the Over and Mackintosh paradigm (Yeterian, 1975; Yeterian, Waters, \& Wilson, 1976), the functional integrity of posterior neocortical areas was shown to be essential to such transfer. Studies of the effects of environmental modifications on brain structure (e.g., Rosenzweig, 1966) and function (e.g., Mailloux, Edwards, Barry, Rowsell, \& Achorn, 1974) have shown that neocortex within the posterior region is affected differentially by varying the content of early environment. With this in mind, we began to explore the effect on CMT of early environments differing in the richness of visual and auditory stimulation.

\section{METHODS}

\section{Subjects and Rearing}

The subjects were 23 male black-hooded rats (Long-Evans derived) obtained from the breeding colony of the University of Connecticut Department of Psychology. They were maintained on a $12 / 12$ reversed day-night cycle until weaning, at which time they were divided into two groups by the split-litter technique: EC $(n=11)$, reared in an enriched environment, and IC $(n=12)$,

This research was supported in part by USPHS Grant MH 10972 to the second author. The present address of the senior author is Harvard Neurological Unit, Beth Israel Hospital, 330 Brookline Avenue, Boston, Massachusetts 02215. Reprint requests should be sent to Edward $\mathrm{H}$. Yeterian, Harvard Neurological Unit, Beth Israel Hospital, 330 Brookline Avenue, Boston, Massachusetts 02215. maintained in an impoverished environment. EC and IC groups were kept in separate rooms, with animals housed in individual cages, $17.8 \times 25.4 \times 17.8 \mathrm{~cm}$. All subjects were given laboratory chow (Purina) and water ad lib, with food and water supplies replenished at the same time for both groups.

EC animals received visual and auditory stimulation daily from 8:00 p.m. to 8:00 a.m.; from 8:00 a.m. to 8:00 p.m., no programmed stimulation from either modality was provided. Visual stimulation was provided by nine $115-\mathrm{V}$ light bulbs of different wattage, from 7.5 to $75 \mathrm{~W}$. The bulbs were mounted in three rows of three each (24-cm center-to-center distance) on an upright plywood panel, $76 \mathrm{~cm}$ in front of the cage rack. Each bulb flashed at an independent and variable rate. Auditory stimulation consisted of line feedback from the lighting panel circuits, amplified by an FM receiver. This variable intensity "static" was emitted from a speaker mounted above, and $44 \mathrm{~cm}$ behind, the lighting panel.

IC animals were kept in a cage rack over which was draped a double thickness of white cloth to provide a diffused visual environment. A $25-\mathrm{W}$ light bulb centered above the rack provided continuous dim illumination. A buzzer on the floor beneath the rack provided unchanging, continuous auditory stimulation.

Both EC and IC animals were kept in their respective environments until they reached 96 days of age. At this time, all were moved to a common room, in which they were maintained throughout training and testing, under continuous lighting. Normal laboratory noises were present outside this room for several hours each day.

\section{Apparatus and Procedure}

The apparatus and procedure were essentially as in Yeterian et al. (1976) and Yeterian (1975). Briefly, a $25 \times 25 \times 30 \mathrm{~cm}$ chamber, placed in a sound-reducing enclosure and controlled by conventional relay equipment, was used. A $9-\mathrm{cm}$ lever mounted directly above a foodcup served as the manipulandum. The only illumination was provided by incandescent bulbs mounted above a white translucent Plexiglas roof. Variations in their intensity gave the discriminative visual stimuli of 14 and $.05 \mathrm{fc}$, respectively, measured $5 \mathrm{~cm}$ above the floor. During preliminary training and auditory transfer, the light was at .6 fc. The auditory stimuli were two intensities ( 96 and $66 \mathrm{~dB}$ SPL) of white noise played through a loudspeaker in the enclosure. During preliminary and visual training, the noise was at $82 \mathrm{~dB}$. 


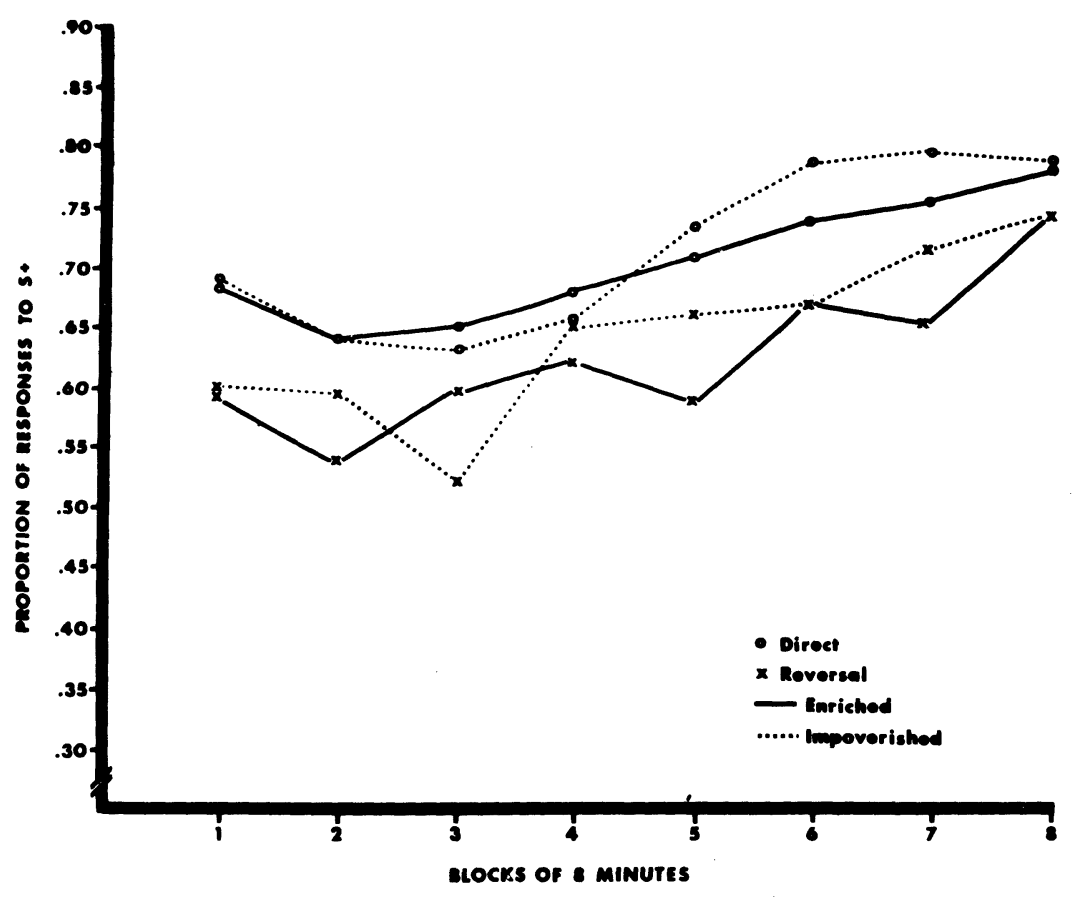

Figure 1. Mean proportions of St responses for the direct and reversal transfer subgroups of enriched and impoverished animals, for 8-min intervals on the auditory transfer session.

With the light and the sound at middle intensities, the rats were pretrained to press the lever for $45-\mathrm{mg}$ food pellets (Noyes), first on a continuous reinforcement schedule, and then on a sequence of variable interval schedules. When all rats had completed pretraining, they then received training on a visual intensity discrimination. The daily sessions were $64 \mathrm{~min}$ long and consisted of presentations of a positive $(\mathrm{S}+)$ or a negative (S-) stimulus. In each of eight 8-min blocks, there were, for each stimulus, two periods of $60 \mathrm{sec}$ and one each of 30 and $90 \mathrm{sec}$; + and $S-$ intervals were balanced within and across sessions. During St, leverpressing was rewarded on a VI 24-sec schedule; during $\mathrm{S}-$, no reinforcement could be gained. For approximately half of the animals in each group, the high intensity was S+ and the low intensity S-. For the other half, the opposite contingencies were used. Training was continued until a given subject's response rate during $\mathrm{S}+$ was at least six times that during $\mathrm{S}$ - for one session. It should be remembered that the middle auditory intensity was maintained throughout the sessions of visual training. On the day immediately following the completion of visual training, each animal received one session of an auditory discrimination, with high intensity $(95 \mathrm{~dB})$ as $\mathbf{S}+$ and low $(66 \mathrm{~dB})$ as $S-$ for all animals. Thus, within each group, half of the animals underwent "direct transfer" (S+ high in both modalities), while the other half underwent "reversal transfer" ( $S+$ low in vision, but high in audition). The sequence of $S+$ and $S-$ stimuli and the schedule of reinforcement on the auditory task followed the same pattern as in visual training.

\section{RESULTS}

EC animals took a mean of 7.2 days to complete all phases of pretraining; IC animals took 5.8 days. The range of days to criterion was 4 to 11 for both groups. On the visual discrimination, EC animals reached criterion in a mean of 10.1 days; IC animals, 9.1 days. The range of days for the former was 5 to 18 ; for the latter, 5 to 15 . Clearly, neither the mean days nor the ranges give any hint that the differential rearing condi- tions affected acquisition of either task. Furthermore, there was no significant difference in speed of acquisition between high- and low-intensity S+ animals, either within or between EC and IC groups.

Figure 1 shows the mean proportions of responses that were made during $\mathrm{S}+$ for each 8 -min block of the 1 day of auditory transfer. The direct transfer groups show better performance than the reversal groups, but it is obvious that no difference arises as a result of rearing conditions. The curves are quite similar to those obtained in previous experiments (e.g., Yeterian et al., 1976) for direct and reversal animals transferred to an auditory discrimination problem with the high intensity as S+. A two-factor analysis of variance, Direct vs. Reversal Transfer by Blocks, with repeated measures on the latter factor, was run (limited, as in previous studies, to the first half of the day). With the groups collapsed across the experience variable, the difference is significant between direct and reversal animals $(F=4.36$, $\mathrm{df}=1 / 21, \mathrm{p}<.05)$. Also, there is a significant difference in responses to $S+$ by animals across the first four blocks of auditory transfer $(\mathrm{F}=2.84, \mathrm{df}=3 / 63$, $\mathrm{p}<.05$ ), suggesting rapid learning in the new modality regardless of direction of transfer or rearing condition. The interaction was not significant.

\section{DISCUSSION}

The results demonstrate that rats reared with a limited amount of visual and auditory stimulation showed as much visual-auditory CMT as rats given an excessive amount of such stimulation. Furthermore, the enriched and impoverished conditions did not affect overall learning ability as measured by the speed of acquisition of the leverpress habit or the visual discrimination. Three lines of explanation for these results may be considered: (1) In many studies demonstrating behavioral or 
anatomical differences related to early experience (e.g., Mailloux et al., 1974), variations in intensity of simple visual and auditory stimuli have been used in conjunction with stimulus objects placed in the animals' home cages. Manipulable objects together with varying ambient stimulation may form an essential combination of environmental factors which results in behavioral effects (dependent on brain modification) that neither factor alone could produce. (2) It is possible that, while the present experimental environments indeed may have resulted in morphological changes, the paradigm was not sufficiently sensitive to reveal concomitant behavioral effects. It may be that acquisition and transfer based on an animal's ability to detect intensity changes in ambient stimulation are "simpler" than tasks in which the relevant intensity cues are localized in discrete stimulus presentation areas (e.g., as in a Thompson-Bryant apparatus), and therefore are less vulnerable to early environmental influences. (3) An alternative possibility is that no effect was seen because specific CMT is a complex, higher order learning process which is not affected by changes induced in basic sensory capabilities of the animal. Had normal CMT been present in impoverished rats against the background of a within-modality deficit, this possibility would have been more clearly strengthened.

In conclusion, modification of early environment did not result in a deficit either on a cross-modal task or a related visual discrimination problem. It remains to be determined whether the lack of an effect was due to peculiarities of environmental con- ditions or of the training and testing paradigm, or to the resistance to environmental influences of processes underlying specific CMT.

\section{REFERENCES}

Mailloux, J. G., Edwards, H. P., Barry, W. F., Rowsell, H. C., \& AChORN, E. G. Effects of differential rearing on cortical evoked potentials of the albino rat. Journal of Comparative and Physiological Psychology, 1974, 87, 475-480.

Over, R. \& Mackintosh, N. Cross-modal transfer of intensity discrimination by rats. Nature, 1969, 224, 918-919.

Rosenweig, M. R. Environmental complexity, cerebral change and behavior. American Psychologist, 1966, 21, 321-332.

YETERIAN, E. H. The effect of visual or auditory cortical lesions on specific cross-modal transfer in the rat. Unpublished doctoral dissertation, University of Connecticut, 1975.

Yeterian, E. H., Waters, R. S., \& Wilson, W. A., JR. Posterior cortical lesions and specific cross-modal transfer in the rat. Physiological Psychology, 1976, in press.

(Received for publication March 4, 1976.) 